\title{
Video Article \\ Improved Visualization of Lung Metastases at Single Cell Resolution in Mice by Combined In-situ Perfusion of Lung Tissue and X-Gal Staining of lacZ- Tagged Tumor Cells
}

\author{
Matthias J.E. Arlt ${ }^{1}$, Walter Born ${ }^{1}$, Bruno Fuchs ${ }^{1}$ \\ ${ }^{1}$ Laboratory for Orthopedic Research, Balgrist University Hospital, Zurich \\ Correspondence to: Matthias J.E. Arlt at marlt@research.balgrist.ch
}

URL: https://www.jove.com/video/4162

DOI: doi:10.3791/4162

Keywords: Cancer Biology, Issue 66, Medicine, Molecular Biology, Cellular Biology, lung metastasis, lacZ-tagging, 5-Bromo-4-chloro-3-indolyl-betaD-galactoside (X-Gal) staining, in-situ lung perfusion, metastases, imaging

Date Published: 8/21/2012

Citation: Arlt, M.J., Born, W., Fuchs, B. Improved Visualization of Lung Metastases at Single Cell Resolution in Mice by Combined In-situ Perfusion of Lung Tissue and X-Gal Staining of lacZ-Tagged Tumor Cells. J. Vis. Exp. (66), e4162, doi:10.3791/4162 (2012).

\section{Abstract}

Metastasis is the main cause of death in the majority of cancer types and consequently a main focus in cancer research. However, the detection of micrometastases by radiologic imaging and the success in their therapeutic eradication remain limited.

While animal models have proven to be invaluable tools for cancer research ${ }^{1}$, the monitoring/visualization of micrometastases remains a challenge and inaccurate evaluation of metastatic spread in preclinical studies potentially leads to disappointing results in clinical trials ${ }^{2}$. Consequently, there is great interest in refining the methods to finally allow reproducible and reliable detection of metastases down to the single cell level in normal tissue. The main focus therefore is on techniques, which allow the detection of tumor cells in vivo, like micro-computer tomography (micro-CT), positron emission tomography (PET), bioluminescence or fluorescence imaging ${ }^{3,4}$. We are currently optimizing these techniques for in vivo monitoring of primary tumor growth and metastasis in different osteosarcoma models. Some of these techniques can also be used for ex vivo analysis of metastasis beside classical methods like qPCR ${ }^{5}$, FACS $^{6}$ or different types of histological staining. As a benchmark, we have established in the present study the stable transfection or transduction of tumor cells with the lacZ gene encoding the bacterial enzyme $\beta$-galactosidase that metabolizes the chromogenic substrate 5-bromo-4-chloro-3-indolyl-beta-D-galactopyranoside (X-Gal) to an insoluble indigo blue dye ${ }^{7}$ and allows highly sensitive and selective histochemical blue staining of tumor cells in mouse tissue ex vivo down to the single cell level as shown here. This is a low-cost and not equipment-intensive tool, which allows precise validation of metastasis ${ }^{8}$ in studies assessing new anticancer therapies ${ }^{9-11}$. A limiting factor of X-gal staining is the low contrast to e.g. blood-related red staining of well vascularized tissues. In lung tissue this problem can be solved by in-situ lung perfusion, a technique that was recently established by Borsig et al. ${ }^{12}$ who perfused the lungs of mice under anesthesia to clear them from blood and to fix and embed them in-situ under inflation through the trachea. This method prevents also the collapse of the lung and thereby maintains the morphology of functional lung alveoli, which improves the quality of the tissue for histological analysis. In the present study, we describe a new protocol, which takes advantage of a combination of X-gal staining of lacZ-expressing tumor cells and in-situ perfusion and fixation of lung tissue. This refined protocol allows high-sensitivity detection of single metastatic cells in the lung and enabled us in a recent study to detect "dormant" lung micrometastases in a mouse model ${ }^{13}$, which was originally described to be non-metastatic ${ }^{14}$.

\section{Video Link}

The video component of this article can be found at https://www.jove.com/video/4162/

Protocol

\section{In-situ Lung Perfusion and Fixation}

1. Anesthetize mice by i.p. injection of $\sim 150 \mu$ l phosphate buffered saline (PBS) containing $112.5 \mathrm{mg} / \mathrm{kg}$ (body weight) ketamine, $16.5 \mathrm{mg} / \mathrm{kg}$ xylazine, and $15 \mathrm{mg} / \mathrm{kg}$ acepromazine (or by another anesthesia containing appropriate pain killers).

2. When reflexes of the mouse are no longer observed, fix it on the operating table back-down and wet the fur with $70 \%$ Ethanol to slick the hairs down.

3. Open the skin from the abdomen up to the neck and pull it to the sides or remove it.

4. Open the peritoneum and the thorax to an ample size. Prevent ruptures of big vessels (e.g. the vena jugularis) to avoid reduced efficiency of the subsequent perfusion.

5. Cut the vena cava caudalis underneath the liver

6. Use a $20 \mathrm{ml}$ syringe equipped with a $21 \mathrm{G}$ - 24G needle to inject slowly $10-15 \mathrm{ml}$ PBS into the right ventricle of the beating heart until the lung is completely white and the heart stops beating (asystole).

7. Pinch off the blood vessels above the liver and the diaphragm with a vascular clamp.

8. Use a $10 \mathrm{ml}$ syringe equipped with a $21 \mathrm{G}-24 \mathrm{G}$ needle to inject $\sim 2 \mathrm{ml}$ of $3 \%$ paraformaldehyde (PFA) in PBS into the right ventricle. 
9. Uncover the trachea outside of the thorax. Use the same syringe to inject $\sim 3 \mathrm{ml}$ of $3 \%$ PFA in PBS cranial (outside of thorax) into the trachea until the lung is inflated. Immediately after removal of the needle, pinch off the trachea caudal of the puncture with a vascular clamp and wait for $10 \mathrm{~min}$ to allow fixation.

10. Transect the blood vessels above the vascular clamp, remove the clamp and inject $\sim 2 \mathrm{ml}$ PBS into the right ventricle to remove excess PFA.

11. Puncture the lower parts of all lung lobes with a needle, remove the vascular clamp at the trachea and inject 3-5 ml of a solution of PolyFreeze embedding medium/PBS 1:1 into the trachea caudal of the first puncture until the PFA in the lung lobes is replaced by the solution.

12. Remove the lung carefully by pulling the heart with a forceps and by transecting connective tissue ligaments, blood vessels and the trachea. Keep the heart connected to the lungs.

13. Depending on how to proceed with the lung tissue analysis continue with the protocols outlined in sections 2 or 3 . If you want to do both analyses, remove one lung lobe and proceed as described under 3. Process the remaining lobes connected to the heart as described under

\section{Visualization of lacZ-tagged Metastatic Tumor Cells in Entire Lung Lobes}

1. Place the lung in a plastic cup with tight closing lid and fix it with $2 \%$ formaldehyde in PBS at room temperature for $30-60$ min.

2. Remove fixation solution and wash thoroughly 3 times with PBS.

3. Add at least $10 \mathrm{ml}$ freshly prepared $5-$-Bromo-4-chloro-3-indolyl- $\beta$-D-galactoside (X-Gal) staining solution (X-Gal stock solution [40 $\mathrm{mg} / \mathrm{ml}$ in Dimethylformamide] 1:40 diluted in Basic Staining Solution; $\mathrm{pH} 7.1$ (Table 1); protect from light).

4. Put a piece of gauze on top of the swimming lung to keep it completely in the solution and place the lid only loosely on the pot to allow exchange of air.

5. Incubate at $37^{\circ} \mathrm{C}$ for $3-5 \mathrm{hr}$ protected from light.

6. Remove X-Gal solution, rinse once with PBS to remove residual X-Gal solution (optional) and add 4\% PFA.

\section{Visualization of lacZ-tagged Metastatic Tumor Cells in Lung Cryosections}

1. Prefill a labeled embedding mold to $1 / 3$ with undiluted PolyFreeze embedding medium. Put the lung lobe on top and fill up with embedding medium until the lobe is completely covered. Try to prevent bubbles.

2. Incubate the embedded lungs at $4{ }^{\circ} \mathrm{C}$ for 20 min.

3. Then freeze the embedded lungs slowly in a mixture of dry ice and Isopentane and store at $-80^{\circ} \mathrm{C}$.

4. Cut 7-10 $\mu \mathrm{m}$ cryosections on a cryostat and mount them on SuperFrostPlus microscope slides.

5. Incubate the sections immediately with $X-G a l$ staining solution at $37^{\circ} \mathrm{C}$ for $24 \mathrm{hr}$ in a humidified chamber.

6. Rinse the slides in PBS 2 times for 5 min and then briefly in distilled water.

7. Counter-stain with nuclear fast red for $10-30 \mathrm{sec}$, rinse in distilled water and mount the slides with Immu-Mount.

\section{Representative Results}

Asai et al. reported in the original article on the Dunn-derived LM8 OS mouse model that s.c. primary tumors derived from the parental Dunn cells, different from those derived from the highly metastatic LM8 sub cell line, do not spontaneously form detectable lung metastases in syngeneic $\mathrm{C} 3 \mathrm{H}$ mice ${ }^{14}$. With the here reported techniques, we reinvestigated the formation of metastases in the Dunn/LM8 mouse OS models. We took advantage of stable lacZ-transduced Dunn and LM8 cells and of a protocol for in-situ lung perfusion and fixation in mice. Representative images of perfused and non-perfused lungs of mice subcutaneously injected with lacZ-transduced and non-transduced control Dunn and LM8 cells are shown in Figure 1. In mice injected with control Dunn cells, macroscopic and microscopic metastases remained undetectable in non-perfused and perfused lungs (Figure 1A, i-iv). But, interestingly, in mice injected with Dunn-lacZ cells, X-gal staining revealed blue micrometastatic foci of single cells or small cell clusters $(<0.1 \mathrm{~mm})$ on the surface of non-perfused lungs (Figure 1A, vi). In-situ perfusion and fixation of the lungs further improved the detectability of Dunn-lacZ micrometastases (Figure 1A, viii). However, outgrowth to macroscopic foci was not observed (Figure 1A, v, vii).

In mice injected with control LM8 cells, translucent, barely detectable macrometastatic foci larger than 0.1 mm in diameter were recognized in non-perfused lungs (Figure 1B, i). Perfusion of the lung (Figure 1B, iii) did not improve the detection of the foci. However, in mice injected with LM8-lacZ cells multiple X-Gal stained blue macro- (Figure 1B, $v$ ) and micrometastases (Figure 1B, vi) were detected on the surface of non-perfused organs. Moreover, perfusion of the lungs further improved the detectability of macro- and micrometastases (Figure 1B, vii-viii). Consequently, micro- and macrometastases became visible at a higher density and a larger number, mainly due to the translucency of the perfused tissue in which foci underneath the organ surface became also visible.

An additional histological analysis using cryosections of lung tissue confirmed the improved detectability of lung metastases in mice injected with lacZ-transduced Dunn and LM8 cells. In mice with primary tumors derived from Dunn-lacZ or LM8-lacZ cells, unlike in mice with primary tumors of the respective control cells, micrometastases or even single cell foci were recognized in lung sections (Figure 2). Moreover, macrometastases were also more clearly visible in mice injected with LM8-lacZ cells than in animals injected with the control LM8 cells (Figure 2C, D). 
A
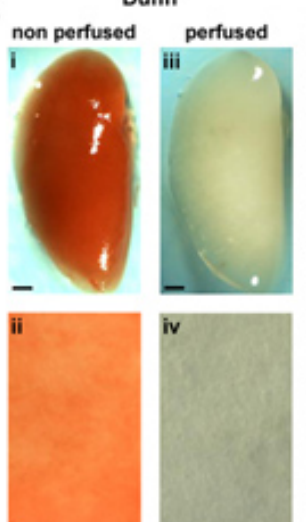

Dunn-lacZ
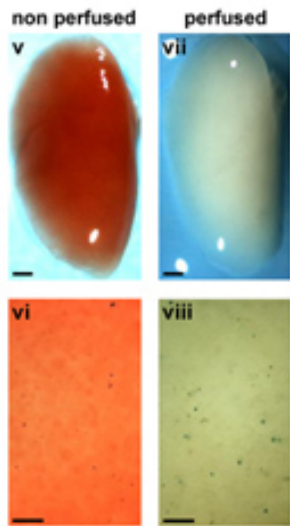

B

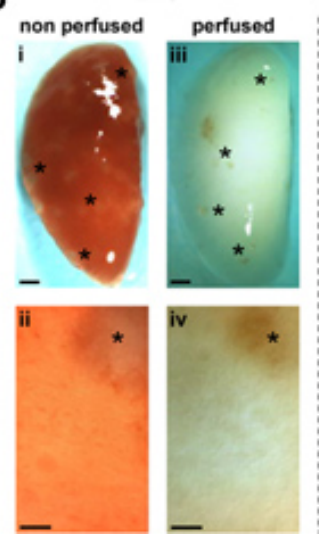

LM8-lacZ

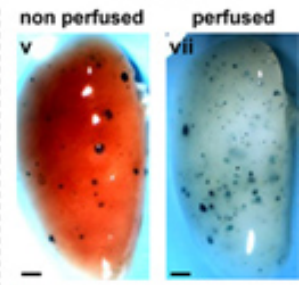

-
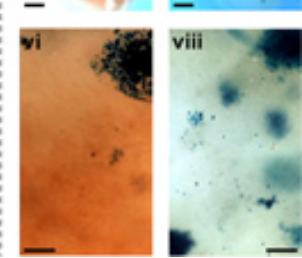

Figure 1. Detection of spontaneous metastases of non-transduced (i-iv) and lacZ-transduced (v-viii) Dunn (A) and LM8 (B) cells in nonperfused (i, ii, v, vi) and perfused (iii, iv, vii, viii) lungs in $\mathrm{C} 3 \mathrm{H}$ mice. Metastases in representative X-Gal-stained whole lungs ( $\mathrm{v}$, vii in $\mathrm{A}$ and $\mathrm{B}$ ) and respective close-ups (vi, viii in A and B) appear blue. Macrometastatic foci $(>0.1 \mathrm{~mm}$ ) in the organs of mice injected with non-tagged LM8 cells (i-iv in B) are indicated by asterisks. Areas shown in close-ups are representative for entire lungs. Scale bars indicate $1 \mathrm{~mm}$ in images of whole lungs and $0.1 \mathrm{~mm}$ in close-ups. Click here to view larger figure.
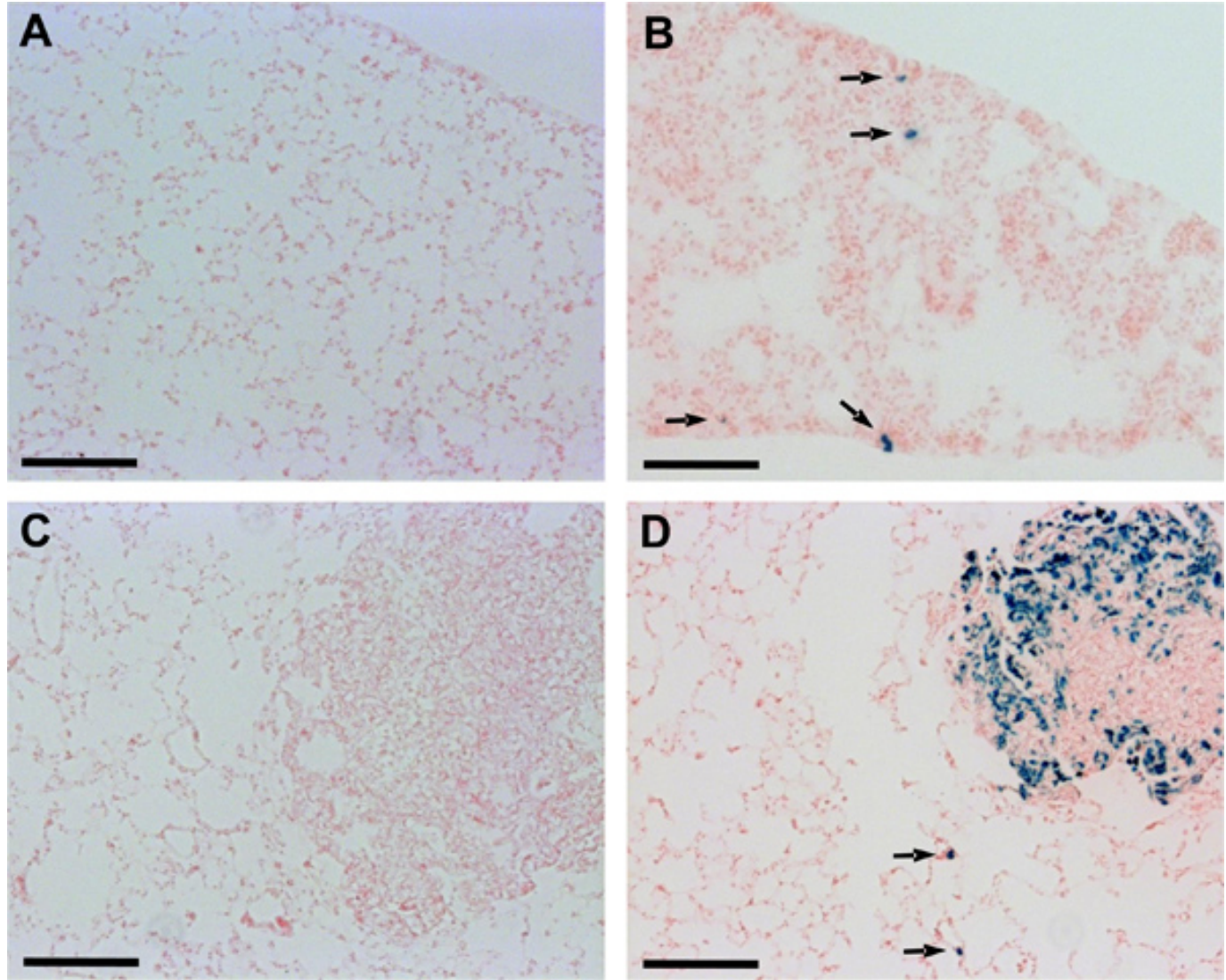

Figure 2. Detection of Dunn and LM8 micrometastases in lung cryosections. Sections of O.C.T. embedded lung tissue were incubated in X-Gal staining solution at $37^{\circ} \mathrm{C}$ for $24 \mathrm{hr}$ in a humidified chamber and then counterstained with nuclear fast red. Arrows point to micrometastatic foci recognized in lung sections of mice injected with Dunn-lacZ (B) or LM8-lacZ cells (D). Micrometastases remained undetectable in lung sections of mice injected with non-transduced Dunn (A) or LM8 cells (C). Scale bars indicate $0.1 \mathrm{~mm}$.

\section{Discussion}

The here presented results in the Dunn/LM8 mouse OS model demonstrate the power of the newly established method that combines the X-Gal staining of lacZ-tagged tumor cells with the in-situ perfusion/fixation of lung tissue. This combination of the two techniques allows high sensitivity detection of micrometastatic lesions down to the single cell level and also improves the visualization of macrometastases on the lung surface (Figure 1) as well as in lung sections (Figure 2). While the X-Gal staining also allows the detection of (micro)metastases in other organs, in-situ perfusion/fixation improves the detectability of metastatic foci in tissues other than the lung only slightly because of the natural blood-and tissuerelated color of these organs ${ }^{13}$. Even if the perfusion is directed to another organ, e.g. the liver, removal of the blood would only partially improve 
the contrast between X-Gal staining and the natural color of the organ. However, the method is applicable to any type of lacZ-tagged tumor cells, will dramatically improve the detectability of lung metastasis down to the level of dormant single-cell micrometastases and enables an easy and reliable quantification of macro- and micrometastases. A limitation of this method and all other techniques that are based on reporter genes, including luciferase and fluorescent proteins, is the stability of the transgene expression. As shown in Figure 2d, not all tumor cells within the macrometastatic foci are stained blue, indicating a lack of beta-galactosidase activity. This might be related to necrosis, but it is more likely due to a loss of transgene expression. We observed that the Dunn and LM8 cells are very effective in the down-regulation of lac $Z$ and other transgenes even under continuous selection for expression. We therefore switched in recent studies to the murine K12 and K7M2 and the human HOS, 143B and SaOS-2 osteosarcoma cell lines, which all maintained stable lacZ expression in vitro as well as in vivo up to $100 \%$ over time.

Once stable lacZ-expression is guaranteed, this technique can be applied in studies with gene-manipulated tumor cells, e.g. to mechanistically investigate the process of tissue colonization ${ }^{13}$ as well as for the development and testing of new therapies aiming at eradication of metastatic lesions ${ }^{15,16}$. Furthermore, it can serve as a benchmark for the improvement of current radiological imaging techniques, such as PET, (micro)CT and MRI, used for early detection of metastatic lesions. In a recent PET study (unpublished) with different tracers we verified the in vivo detected lung metastases subsequently ex vivo with the described protocol. In an ongoing study with a new small animal micro-CT (SkyScan) we are so far able to detect lung metastases in vivo down to a size of $0.5 \mathrm{~mm}$ and ex vivo down to $0.3 \mathrm{~mm}$, but we aim at a resolution of $0.1 \mathrm{~mm}$. Interestingly, this is the size limit that we set to distinguish macro- from micrometastases with the combined method of in-situ perfusion and X-Gal staining. This underlines again the sensitivity and usefulness of this easy and cost-effective technique.

\section{Disclosures}

No conflicts of interest declared.

\section{Acknowledgements}

The authors would like to thank Dr. Lubor Borsig (Institute of Physiology, University of Zurich) for his advice on the technique of lung perfusion. This work was supported by grants from the Krebsliga of the Kanton Zurich, the Walter L. and Johanna Wolf Foundation, Zurich, the Lydia Hochstrasser Foundation, Zurich, the Swiss National Science Foundation, SNF, Switzerland, the Schweizerischer Verein Balgrist, and the University of Zurich.

\section{References}

1. Frese, K.K. \& Tuveson, D.A. Maximizing mouse cancer models. Nature reviews. 7, 645-658, doi:10.1038/nrc2192 [pii] nrc2192 (2007).

2. Coussens, L.M., Fingleton, B., \& Matrisian, L.M. Matrix metalloproteinase inhibitors and cancer: trials and tribulations. Science (New York, N.Y.) 295, 2387-2392 (2002).

3. Fass, L. Imaging and cancer: a review. Mol Oncol 2, 115-152, doi:10.1016/j.molonc.2008.04.001 [pii] S1574-7891(08)00059-8 (2008).

4. Puaux, A.L., et al. A comparison of imaging techniques to monitor tumor growth and cancer progression in living animals. Int. J. Mol. Imaging. 2011, 321538, doi:10.1155/2011/321538 (2011).

5. Malek, A., Catapano, C.V., Czubayko, F., \& Aigner, A. A sensitive polymerase chain reaction-based method for detection and quantification of metastasis in human xenograft mouse models. Clinical \& experimental metastasis. 27, 261-271, doi:10.1007/s10585-010-9324-1 (2010).

6. Schmidt, C.M., et al. Characterization of spontaneous metastasis in an aggressive breast carcinoma model using flow cytometry. Clinical \& experimental metastasis. 17, 537-544 (1999).

7. Horwitz, J.P., et al. Substrates for Cytochemical Demonstration of Enzyme Activity. I. Some Substituted 3-Indolyl-Beta-D-Glycopyranosides. J. Med. Chem. 7, 574-575 (1964).

8. Kruger, A., Schirrmacher, V., \& Khokha, R. The bacterial lacZ gene: an important tool for metastasis research and evaluation of new cancer therapies. Cancer metastasis reviews. 17, 285-294 (1998).

9. Arlt, M., et al. Increase in gelatinase-specificity of matrix metalloproteinase inhibitors correlates with antimetastatic efficacy in a T-cell lymphoma model. Cancer research. 62, 5543-5550 (2002).

10. Arlt, M.J., et al. Efficient inhibition of intra-peritoneal tumor growth and dissemination of human ovarian carcinoma cells in nude mice by antiL1-cell adhesion molecule monoclonal antibody treatment. Cancer research. 66, 936-943 (2006).

11. Banke, I.J., et al. Effective inhibition of experimental metastasis and prolongation of survival in mice by a potent factor Xa-specific synthetic serine protease inhibitor with weak anticoagulant activity. Thrombosis and haemostasis. 94, 1084-1093 (2005).

12. Borsig, L., et al. Heparin and cancer revisited: mechanistic connections involving platelets, P-selectin, carcinoma mucins, and tumor metastasis. Proceedings of the National Academy of Sciences of the United States of America. 98, 3352-3357 (2001).

13. Arlt, M.J., et al. LacZ transgene expression in the subcutaneous Dunn/LM8 osteosarcoma mouse model allows for the identification of micrometastasis. J. Orthop. Res. 29, 938-946, doi:10.1002/jor.21304 (2011).

14. Asai, T., et al. Establishment and characterization of a murine osteosarcoma cell line (LM8) with high metastatic potential to the lung. International journal of cancer. 76, 418-422 (1998).

15. Arlt, M. J., et al. The antineoplastic antibiotic taurolidine promotes lung and liver metastasis in two syngeneic osteosarcoma mouse models and exhibits severe liver toxicity. International journal of cancer. doi:10.1002/ijc.27378 (2011).

16. Steinmann, P., et al. Antimetastatic activity of honokiol in osteosarcoma. Cancer. doi:10.1002/cncr.26434 (2011). 